\title{
Diretrizes para implantação de sistemas de segurança e saúde do trabalho em empresas produtoras de baterias automotivas
}

\author{
Guidelines to implement occupational health and \\ safety systems in automotive batteries manufacturers
}

\author{
Otávio José de Oliveira ${ }^{1}$ \\ Alessandra Bizan de Oliveira ${ }^{1}$ \\ Renan Augusto de Almeida ${ }^{1}$
}

\begin{abstract}
Resumo: O desenvolvimento de Sistemas de Gestão da Segurança e Saúde no Trabalho (SGSST) ganha um significado cada vez mais importante no desempenho das empresas, pois, por meio deles, é possível obter a promoção da saúde e satisfação dos trabalhadores e a redução dos riscos de acidentes. No entanto, para que um SGSST obtenha bons resultados, as empresas precisam estar atentas às dificuldades comumente encontradas durante o seu processo de implantação, procurando solucioná-las de maneira antecipada e estruturada. Pelo exposto, este trabalho tem como principal objetivo apresentar diretrizes, baseadas no referencial teórico e nos resultados dos estudos de caso realizados, para implantação de SGSSTs em empresas fabricantes de baterias automotivas. Para o seu desenvolvimento adotou-se o método de pesquisa qualitativa a partir da realização de dois estudos de caso em empresas fabricantes de baterias automotivas localizadas na cidade de Bauru. Os instrumentos de coleta de dados foram entrevistas semiestruturadas, análise de documentos e observação in loco. Ao final do artigo, são propostas diretrizes relacionadas aos seguintes elementos: alta direção, estratégia organizacional, cultura organizacional, departamento de Segurança e Saúde do Trabalho (SST), técnicos de SST, recursos humanos, treinamento, equipes multidisciplinares, comunicação interna, resistência à mudança, indicadores de desempenho, ferramentas gerenciais para solução de problemas de SST, gestão de projetos, recompensas e incentivos, e integração do sistema.
\end{abstract}

Palavras-chave: Sistema de gestão da segurança e saúde no trabalho. Implantação de sistemas de gestão. Setor de baterias automotivas. Resistência à mudança.

\begin{abstract}
The development of Health and Safety Management Systems has become increasingly important for the performance of companies since they can provide workers with good health and satisfaction and reduce the risks of accidents. However, in order for these systems to be efficient, the organizations need to be aware of the difficulties found during their implementation to overcome them ahead in a structured way. The main objective of this paper is to present guidelines based on theoretical basis and on the results of case studies to implement Occupational Health and Safety Management Systems in automotive battery manufacturers. A qualitative case study research was carried out in two battery automotive manufacturing companies located in the city of Bauru, state of São Paulo. The data were collected through semi-structured interviews, analysis of documents, and observation in loco. Guidelines related to the following elements were proposed for the implementation of this kind of system : top administration, organizational strategy, organizational culture, Occupational Health and Safety Department (OHSD), Occupational Safety and Health technicians, human resources, training, multidisciplinary teams, internal communication, resistance to changes, performance indicators, management tools for problem solution, projects management, rewards and incentives, and system integration.
\end{abstract}

Keywords: Occupational health and safety management system. Management system implementation. Automotive battery manufacturer. Resistance to changes.

\footnotetext{
${ }^{1}$ Universidade Estadual Paulista - UNESP, Av. Eng. ${ }^{\circ}$ Luiz Edmundo Carrijo Coube, 14-01, Vargem Limpa,

CEP 17033-360, Bauru - SP, Brasil, E-mails: otavio@ feb.unesp.br; alebizan@terra.com.br; renan_aa@ feb.unesp.br
}

Recebido em 29/12/2008 — Aceito em 28/4/2010

Suporte financeiro: FAPESP. 


\section{Introdução}

As recentes mudanças econômicas, sociais e tecnológicas impõem às organizações uma busca incessante por novas estratégias e ferramentas gerenciais para auxiliá-las na melhoria de seus processos e produtos. Neste sentido, elas têm desenvolvido/ implantado sistemas de gestão que aumentem a qualidade de seus produtos e serviços, possibilitem o desenvolvimento sustentável, melhorem as condições de vida de seus colaboradores e, consequentemente, incrementem sua lucratividade e competitividade, transformando, dessa forma, as pressões de mercado em vantagens competitivas.

É neste cenário que os Sistemas de Gestão da Segurança e Saúde do Trabalho (SGSST) têm sido cada vez mais objeto de atenção dos gestores, que os têm adotado como forma de gerar vantagens em relação à concorrência.

Os SGSSTs, a partir do desenvolvimento de ferramentas para detecção e redução do risco de acidentes do trabalho, proporcionam contínua melhoria, racionalização e confiabilidade de projetos, processos e produtos/serviços, proporcionando redução de acidentes e doenças ocupacionais, minimização dos custos de processos, motivação e aumento da qualidade de vida dos trabalhadores, melhoria da imagem da organização e incremento da sua competitividade e lucratividade.

A perspectiva destes resultados torna-se ainda mais relevante ao se considerar o setor de baterias automotivas, objeto de estudo deste trabalho, em virtude de sua relevância econômica e de suas características peculiares, como a manipulação em grande escala e intensiva de elementos químicos nocivos à saúde humana.

Este setor é responsável pelo consumo de grandes quantidades de chumbo e, embora seus efeitos tóxicos sejam bastante conhecidos e diversas doenças estejam associadas à contaminação por esse metal, evidências sugerem que altas exposições ao chumbo continuam a ser um dos maiores problemas de saúde pública e ocupacional (MATTOS et al., 2003).

Porém, assim como com qualquer outro novo sistema, sua implantação esbarra em uma série de dificuldades, que podem ser evitadas ou amenizadas se consideradas de forma antecipada quando do seu planejamento e se utilizados os instrumentos e técnicas disponíveis para sua mitigação.

Portanto, a questão que norteou o desenvolvimento desta pesquisa foi: quais práticas podem reduzir as dificuldades de implantação de SGSSTs e potencializar seus resultados?

Em face do exposto, este artigo tem como principal objetivo apresentar diretrizes, baseadas no referencial teórico e nos resultados de dois estudos de caso, para implantação de SGSSTs em empresas fabricantes de baterias automotivas.

Para garantir seu foco, procurou-se delimitar o escopo da pesquisa em relação aos seguintes elementos: objeto de estudo (Sistemas de Gestão da Segurança e Saúde no Trabalho), recorte geográfico (cidade de Bauru - centro-oeste paulista) e segmento de atuação das empresas (setor de baterias automotivas).

Para embasar a realização do trabalho, tendo-se em vista a questão de pesquisa posta e o objetivo proposto, é apresentada uma revisão teórica sintetizada sobre Sistemas de Gestão da Segurança e Saúde no Trabalho, gestão de mudanças e ferramentas e técnicas de apoio à implantação de SGSSTs.

\section{Método de pesquisa}

A realização deste trabalho se deu por meio de uma pesquisa qualitativa com base na metodologia de estudo de caso duplo. Segundo Santos, Rossi e Jardilino (2000), este tipo de pesquisa se preocupa fundamentalmente com a compreensão e interpretação do fenômeno estudado, tendo como principal objetivo compreendê-lo, explorá-lo e especificá-lo. Esta escolha, segundo Yin (2003), permite que se obtenham conclusões analíticas e, consequentemente, se contribua com a teoria vigente, como no caso das diretrizes apresentadas neste trabalho.

A escolha das empresas se deu principalmente devido à importância da SST em seu tipo de atividade e pela relevante contribuição que seu estudo poderia dar à formulação das diretrizes aqui propostas. Mas também cabe destacar que a facilidade de acesso às informações e a permissão para visitar suas plantas industriais contribuíram para esta decisão. Os instrumentos de coleta de dados utilizados para sua realização foram observação in loco (visitas às plantas industriais e à sala de reuniões das CIPAS), análise de documentos (procedimentos, instruções de trabalho, registros, etc.) e entrevistas semiestruturadas com os técnicos de segurança, gerentes de produção e alguns funcionários-chave do chão de fábrica, conforme indicado por Cervo e Bervian (2002), Lakatos e Marconi (2002) e Yin (2003).

Ressalta-se que, apesar da permissão para acesso às instalações físicas e consulta aos documentos de SST, encontrou-se muita resistência por parte, principalmente, dos gestores, das duas empresas pesquisadas. Não foi permitida a divulgação dos poucos dados ou indicadores mais diretamente relacionados à SST existentes. Também não foi autorizado à equipe de pesquisadores fotografar ou reproduzir em forma de desenho o layout da empresa ou mesmo parte dele.

As diretrizes propostas neste trabalho foram elaboradas com base no referencial teórico e com base nos referidos estudos de caso, a partir da triangulação entre as informações obtidas com as três fontes de evidências citadas.

Na Figura 1 apresenta-se o esquema da método científico utilizada nesta pesquisa. 


\section{Sistemas de Gestão da Segurança e Saúde no Trabalho}

Alguns trabalhos relevantes (QUELHAS; ALVES; FILHARDO, 2003; OLIVEIRA, 2003; HASLE; JENSEN, 2006; LOOSEMORE; ANDONAKIS, 2007; CHOUDHRY; FANG; MOHAMED, 2007; AKSORN; HADIKUSUMO, 2008; DUIJM et al., 2008) destacam a importância dos SGSSTs para as organizações, evidenciando seus aspectos preventivos e os benefícios que podem proporcionar.

O processo de implantação de um sistema de gestão agrega valor à cultura organizacional, pois desenvolve competências relacionadas ao planejamento e execução das atividades, prioriza a capacidade de trabalho em equipe e promove a confiabilidade do sistema produtivo.

O desenvolvimento de SGSSTs tem sido a principal estratégia empresarial para enfrentar o sério problema social e econômico dos acidentes e doenças relacionadas ao trabalho, e ainda pode ser usado pelas empresas como um fator para aumento da competitividade (TRIVELATO, 2002).

Implementar um SGSST traz benefícios como alinhamento das necessidades dos colaboradores com a política e diretrizes de segurança, transmissão de mais confiança para os clientes internos e externos e diminuição da susceptibilidade da empresa em relação aos passivos trabalhistas e de fiscalização. Contudo, para se obter sucesso na implementação desse tipo de sistema, a alta administração deve buscar, por meio de atitudes e recursos, a direta e intensa participação de todos os trabalhadores (PINTO; SÁ, 2007).

As organizações podem padronizar seu SGSST por meio de normas e diretrizes, sendo que a mais conhecida e utilizada é a OHSAS 18001 (Occupational Health and Safety Assessment Series), norma formulada em 1999 por um grupo de entidades internacionais (Bureau Veritas Certification - BVQI, Det Norske Veritas - DNV, Grupo Lloyd's Register - LLOYDS, outras) e publicada pela British Standards Institution (BSI) para atender às necessidades das empresas de todo o mundo com relação ao gerenciamento de suas obrigações de segurança e saúde ocupacional.

Em julho de 2007, a norma OHSAS 18001:1999 foi substituída pela OHSAS 18001:2007 e algumas alterações foram feitas, refletindo a experiência de 16000 organizações certificadas em mais de 80 países (QSP, 2007).

A Figura 2 indica a macroestrutura da norma OHSAS 18001, que é baseada na metodologia PDCA (plan, do, check e action) (OHSAS 18001, 2007).

\section{Gestão da mudança}

As organizações têm alcançado inúmeros benefícios com a implantação de SGSSTs. Porém, neste processo surgem dificuldades que podem reduzir

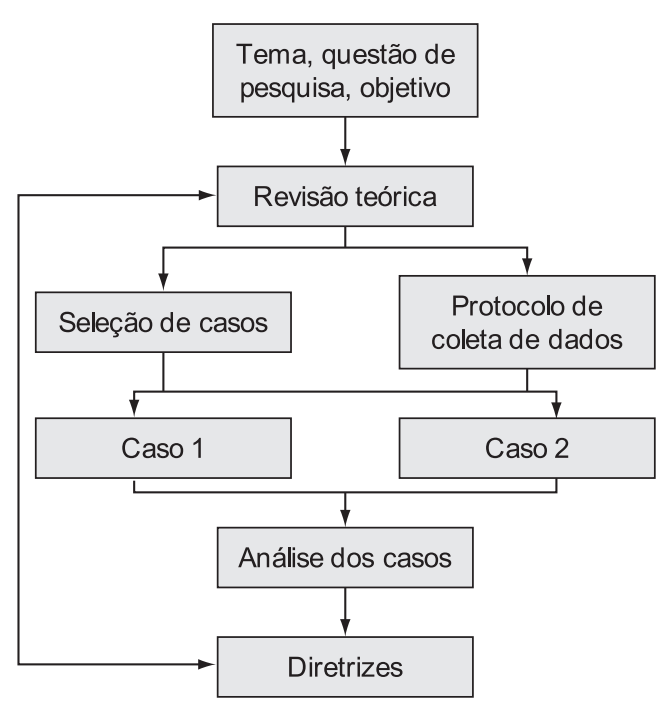

Figura 1. Estrutura metodológica da pesquisa.

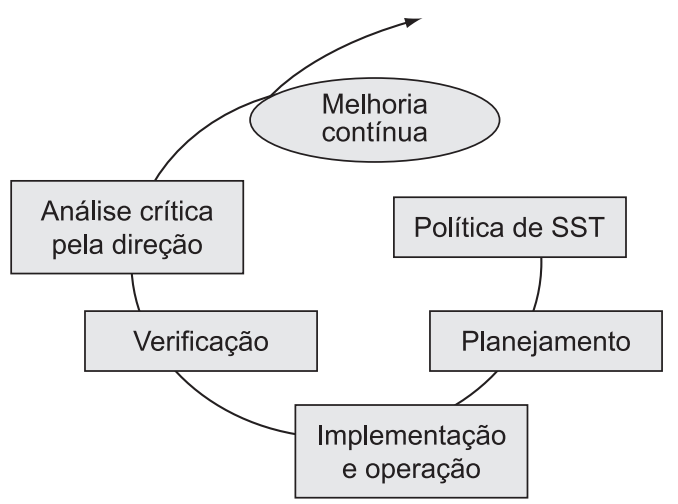

Figura 2. Espiral do sistema de segurança e saúde no trabalho. Fonte: British Standards Instituition (2007).

seus potenciais resultados. A sua eficiência poderia ser consideravelmente maior se fossem previamente observados alguns fatores negativos e de comum ocorrência, como perfil e falta de experiência dos empresários neste assunto, indicadores de desempenho focados apenas nos aspectos financeiros, falta de constância de propósito, alegação de falta de tempo para a realização de algumas tarefas de implantação, dificuldade em estabelecer metas e planos estratégicos a longo prazo, falta de sentimento dos funcionários como efetivos contribuidores para o crescimento da empresa, documentos mais burocráticos do que o necessário, alta rotatividade da força de trabalho e pouca utilização de registros (ANHALON; ZOQUI; PINTO, 2005).

O comportamento humano no ambiente de trabalho é um dos itens mais importantes na implantação de novos sistemas e vem sendo amplamente estudado nos últimos tempos, destacando-se, no aspecto emocional, a satisfação que o trabalhador possui em 
sua atividade. Não há um fator único determinante para esta satisfação, ela depende do ambiente e das condições de trabalho, ou seja, da qualidade de vida no trabalho (MARQUEZE; MORENO, 2005; HASLAM et al., 2005).

A resistência à mudança tem sido entendida como a principal causa de conflitos indesejáveis e prejudiciais à organização e prejudica muito a implantação de SGSSTs, porém pode ser minimizada com técnicas apropriadas. A mudança é geralmente temida porque significa perturbação do status quo, ameaça aos direitos adquiridos e transtorno à maneira estabelecida de se realizarem as tarefas. As pessoas afetadas pela mudança experimentam alguma confusão emocional, envolvendo sensação de perda e incerteza. Embora muitas mudanças não cheguem a provocar os efeitos esperados, sua simples expectativa leva as pessoas a resistirem (OLIVEIRA, 2004).

A capacidade dos gestores em desenvolver bons sistemas de gestão também é um elemento determinante para o seu bom desempenho. Quando a qualidade da gestão é ruim, podem ocorrer grandes dificuldades organizacionais, tais como alta rotatividade de profissionais capacitados, ausência de incentivos para a realização das atividades, falhas na comunicação organizacional e distorções nas estruturas de poder.

Segundo Aggelogiannopoulos, Drosinos e Athanasopoulos (2007), a falta de experiência e conhecimento dos funcionários sobre o novo sistema a ser implantado é um importante obstáculo a ser superado. O inadequado fluxo das informações técnicas, de legislações e requisitos aplicáveis ao negócio da empresa, bem como a falta de divulgação dos resultados de desempenho são fatores limitantes para que o processo de implantação de sistemas de gestão alcance bons resultados.

Esta deficiência pode estar relacionada a diversos fatores que vão além das limitações da direção e da gerência, tal como a necessidade de infraestrutura adicional para a integração das informações relativas ao sistema e dificuldades em correlacionar os dados encontrados (SHI et al., 2008).

\section{Ferramentas e técnicas de apoio à implantação de Sistemas de Gestão da Segurança e Saúde no Trabalho}

A globalização e a internacionalização dos mercados têm aumentado a competitividade entre as empresas, o que as têm conduzido a se empenharem em projetos que possam garantir sua sobrevivência, como o desenvolvimento de SGSSTs. Só que estes projetos precisam ser adequadamente planejados, organizados, monitorados, controlados e avaliados, sob pena de não gerarem os resultados esperados (RAYMOND; BERGERON, 2008).
Segundo Chan e Wong (2006), a alta direção tem um papel muito importante na implantação e manutenção destes sistemas, pois, sem o seu compromisso e envolvimento, o programa não ganha credibilidade diante dos colaboradores, o que afeta diretamente a eficácia deste processo.

Os funcionários se tornam mais dispostos a cooperarem com as mudanças propostas quando começam a acreditar no real comprometimento da direção (LANGFORD; ROWLINSON; SAWACHA, 2000). É esta participação conjunta entre direção e colaboradores que propicia o sentimento de responsabilidade mútua, tornando-se um fator decisivo para o sucesso da mudança (CHOUDHRY; FANG; MOHAMED, 2007).

Uma das chaves para o sucesso das organizações está no desenvolvimento de uma cultura corporativa sólida. A alta direção deve construí-la por meio da articulação de um conjunto de valores, que devem ser reforçados por políticas formais e informais, partilhada e respeitada por todos os colaboradores (MILAN; PRETTO; PIGOZZI, 2005). Este processo é um mecanismo poderoso para criar as condições para implantação de um SGSST.

Gerenciar positivamente a cultura organizacional é um grande desafio, porém seu êxito permitirá às empresas melhorarem sua eficácia e maximizarem seu desempenho por meio da identificação das principais competências, habilidades e comportamentos que irão conduzir a um movimento pró-segurança (DINGSDAG; BIGGS; SHEAHAN, 2008).

Rondinelli e Vastag (2000) afirmam que a implantação de novos sistemas exige o planejamento e execução de avaliações sistemáticas e constantes treinamentos. O treinamento é responsável por elevar a performance do capital intelectual e um importante elemento para amenizar a resistência às mudanças.

A área de recursos humanos (RH) tem como fim a utilização das pessoas para o alcance dos objetivos empresariais, reconhecendo-se que a qualidade dessa gestão influencia diretamente na capacidade da organização e de seus empregados em levar a cabo suas incumbências, incluindo-se o auxílio ao desenvolvimento de um SGSST (MILKOVICH; BOUDREAU, 2000).

Segundo Aggelogiannopoulos, Drosinos e Athanasopoulos (2007), a comunicação sobre a implantação de novos sistemas, elemento essencial para o seu sucesso, pode ser realizada por meio de treinamentos internos e externos em que os funcionários têm a oportunidade de se familiarizarem com o sistema e os documentos utilizados, bem como entender as suas responsabilidades individuais e os benefícios derivados deste projeto.

Um sistema de medição deve ser criado e estruturado de tal forma que facilite a análise crítica dos resultados alcançados com a implantação do SGSST e, assim, 
permita uma tomada de decisão com maior confiança e precisão (QUINTELLA; LIMA, 2004).

Definir indicadores de desempenho em segurança do trabalho e retroalimentar o sistema possibilitam redirecionar esforços quando necessários e estimular os colaboradores a se engajarem, de forma cada vez mais intensa, na implementação de melhorias contínuas (GODOY; PEÇANHA, 2007).

\section{Estudos de caso}

As empresas estudadas, denominadas neste trabalho de Empresa 1 e Empresa 2, são fabricantes de baterias automotivas e localizam-se na cidade de Bauru - região centro-oeste do Estado de São Paulo.

A bateria é um conjunto de acumuladores elétricos que possui a propriedade de converter energia química em energia elétrica, tendo como função abastecer o sistema elétrico do veículo de energia e acumulá-la quando o veículo estiver em funcionamento $(\mathrm{BOSCH}$, 2008).

Sua utilização em automóveis surgiu em 1912, sendo, ainda hoje, articulada aos sistemas elétrico e eletrônico dos automóveis, e responsável pela alimentação de seus componentes elétricos (SBRT, 2006). As baterias automotivas comuns possuem a composição típica descrita na Tabela 1.

O chumbo $(\mathrm{Pb})$, principal elemento da composição da bateria, é um metal com uma longa história de uso industrial e com importantes efeitos tóxicos sobre a saúde humana. Estudos epidemiológicos realizados em diversos países do mundo relatam inúmeros casos de intoxicação, notadamente em exposições ocupacionais e ambientais (MENEZES; CARVALHO; SPÍNOLA, 2004).

No ambiente laboral, a absorção do chumbo resulta principalmente da inalação de pequenas partículas de tamanho respirável ou dos fumos do metal. Secundariamente, pode ocorrer absorção por meio da ingestão de alimentos contaminados, água e por hábitos do trabalho, como o de fumar, e por contaminação dérmica e do vestuário.

Os processos de fundição e refinaria são provavelmente, entre todas as modalidades de

Tabela 1. Composição típica das baterias.

\begin{tabular}{cc}
\hline \multicolumn{3}{c}{ Elementos (\%) } \\
\hline $\mathrm{Pb}$ & 71,2 \\
$\mathrm{SO}_{4}$ & 18,1 \\
$\mathrm{Sb}$ & 0,3 \\
$\mathrm{Ca}$ & 0,4 \\
$\mathrm{SiO}_{2}$ & 0,14 \\
$\mathrm{As}$ & $<0,04$ \\
$\mathrm{Sn}$ & $<0,1$ \\
\hline
\end{tabular}

Fonte: CETEM (2003). exposição ocupacional, as que apresentam maior risco de exposição ao metal (MENEZES; CARVALHO; SPÍNOLA, 2004).

A Empresa 1 possui um canal de comercialização que inclui 21 centros de distribuição próprios, 30 centros de distribuição terceirizados no Brasil e mais 20 centros de distribuição internacional na América do Sul, América Central, Ilhas do Caribe, África e Europa. Os principais produtos produzidos são baterias para veículos automotores, baterias estacionárias e tracionárias. Em seu quadro de empregados constam 417 funcionários, sendo 94 trabalhadores do setor administrativo.

É certificada segundo as normas ISO 9001 (Sistema de Gestão da Qualidade) e ISO 14001 (Sistema de Gestão Ambiental), e está em processo de implantação da TS 16949 (Especificação automotiva) e planejamento futuro para a implantação da OHSAS 18001 (Sistema de Gestão da Segurança e Saúde no Trabalho). Atualmente, o seu Sistema de Gestão da Segurança e Saúde no Trabalho se baseia no cumprimento de normas regulamentadoras instituídas pelo Ministério do Trabalho e Emprego Brasileiro (MTE).

Mesmo já possuindo sistemas de gestão conforme ISO 9001 e ISO 14001, a empresa não possui indicadores de desempenho sobre segurança e saúde no trabalho. Os técnicos de segurança têm a intenção de elaborá-los, porém nada está sendo feito para isto. Desta maneira, a evolução dos resultados de SST não são conhecidos, e algumas ações corretivas e preventivas acabam não sendo adotadas após os acidentes. $\mathrm{O}$ objetivo dos indicadores é que eles fiquem constantemente expostos, para que cada empregado conheça os riscos de sua atividade e se sinta responsável na melhoria dos índices.

Vale destacar que um levantamento realizado pela empresa no ano de 2006 revelou que em torno de $44 \%$ dos acidentes ocorridos foram gerados por empregados contratados a menos de um ano. Em função disso, a organização mantém um programa de integração com os novos funcionários, em que, além de divulgar a missão, visão e valores da empresa, mostra a sua história, os produtos fabricados, os clientes, os benefícios, as regras de conduta, os seus direitos e deveres, além de serem dadas orientações sobre medidas de prevenção de acidentes e doenças ocupacionais. Este novo empregado é acompanhado durante os primeiros seis meses de trabalho com maior rigidez que a média, sendo avaliado por seu superior imediato, quanto a seu comportamento em relação às normas internas de segurança da empresa e, caso seja necessário, intervenções são feitas pelos técnicos de segurança no trabalho, para assegurar que o novo funcionário entenda as normas e trabalhe com segurança. 
A Empresa 2 é certificada segundo as normas ISO 9001 (Sistema de Gestão da Qualidade), estando em processo de implantação da ISO 14001 (Sistema de Gestão Ambiental) e planejamento futuro para a implantação da OHSAS 18001 (Sistema de Gestão da Segurança e Saúde no Trabalho). Quanto à segurança e saúde no trabalho, a empresa cumpre as normas regulamentadoras instituídas pelo Ministério do Trabalho e Emprego Brasileiro. A empresa possui aproximadamente 1000 empregados, tanto diretos como indiretos.

A alta direção participa do planejamento e da implantação de ações de segurança e saúde no trabalho, realizando reuniões periódicas, para o acompanhamento dos resultados. Esta é uma prática que dentro da organização traz bons resultados, pois percebendo o envolvimento da Direção com as ações de segurança e saúde no trabalho, os funcionários se sentem motivados a colaborar, sugerindo melhorias e participando da elaboração de novos projetos.

Apesar do envolvimento da alta direção com o sistema de segurança e saúde no trabalho, os objetivos propostos e os benefícios a serem alcançados com a implantação de novos programas de SST não são adequadamente divulgados aos funcionários. Este fato gera conflitos e incertezas, por parte dos funcionários, quanto aos objetivos e resultados esperados pela organização, com relação à segurança e saúde no trabalho e à responsabilidade de cada funcionário neste processo. Alguns funcionários se mostram também resistentes à adoção de novos métodos de trabalho ou mudanças na supervisão.

No Quadro 1 são apresentadas suas principais características à luz da SST.

De modo geral, destaca-se na Empresa 1 o considerável investimento em melhorias na área de segurança e saúde, o apoio dos técnicos de segurança ao desenvolvimento da SST na empresa e o baixo envolvimento da área de RH neste processo.

Uma das grandes reclamações dos técnicos de segurança da empresa é o não atendimento por parte de alguns colaboradores das regras de segurança estabelecidas. Esta resistência, na maioria das vezes, tem início na média gerência, o que acaba gerando conflitos com os demais colaboradores, que também não se acham obrigados a segui-las.

O trabalho dos técnicos de segurança é desenvolvido individualmente com cada colaborador de acordo com o nível de aceitação das normas e dos procedimentos relativos à segurança, procurando sempre desenvolver um programa personalizado para incutir a cultura de segurança nos mais resistentes.

Os resultados referentes à segurança e saúde no trabalho na organização não estão vinculados a nenhum sistema de remuneração ou qualquer outro incentivo, porém existem estudos para que isto possa acontecer no futuro.
Observa-se na Empresa 2 maior eficiência em executar as medidas de SST e no acompanhamento e desenvolvimento de melhorias no SGSST que na Empresa 1. A maioria das atividades é executada pelos técnicos de segurança, contudo observa-se um grande esforço da área de recursos humanos em se envolver nestas atividades, principalmente no que diz respeito aos treinamentos de funcionários.

Verificou-se a existência de documentos importantes para cumprimento e coordenação das atividades de SST. Os principais foram o histórico de segurança, o formulário de solicitação de serviços, identificação dos acidentes por setor, registros de treinamentos, atas de reuniões de segurança, inspeções de melhoria, etc.

A organização possui operadores treinados, equipamentos em bom estado de conservação, procedimentos de manutenção corretiva, preventiva, preditiva, além de um check list, visando à segurança e saúde dos trabalhadores e um ambiente sadio e produtivo.

No Quadro 2 estão destacadas as principais dificuldades relativas à SST nas empresas estudadas.

\section{Diretrizes para implantação de Sistemas de Gestão da Segurança e Saúde no Trabalho em empresas produtoras de baterias automotivas}

Com base no referencial teórico e nos estudos de caso realizados foram elaboradas algumas diretrizes gerais para contribuir com a implantação de SGSSTs em empresas fabricantes de baterias automotivas. São elas:

\subsection{Alta direção}

A alta direção deve estar comprometida com o processo de implantação do SGSST, participando ativamente de seu planejamento, execução e avaliação dos resultados. São os superiores hierárquicos quem devem dar o exemplo e trabalhar incessantemente pela busca de adesões a este processo.

Este comprometimento deve ser rotineiro e se dar por meio da busca de bons resultados, participação em reuniões e comunicação direta com os funcionários sobre as mudanças realizadas e suas consequências no desempenho da organização e nas suas vidas pessoais.

É também de grande importância que seja da alta direção a principal iniciativa de se implantar o SGSST, para que ela participe e lidere ativamente o estabelecimento de metas e esteja consciente do benefício que sua implantação trará para os funcionários e para a empresa como um todo. 
Quadro 1. Resumo das principais práticas de segurança e saúde no trabalho nas empresas estudadas.

\begin{tabular}{|c|c|c|}
\hline \multicolumn{3}{|c|}{ Principais características de SST nas empresas estudadas } \\
\hline & Empresa 1 & Empresa 2 \\
\hline $\mathrm{N}^{\circ}$ Funcionários & 417 & 362 \\
\hline Composição do SESMT & 1 Engenheiro de segurança & 1 Enfermeira do trabalho \\
\hline (Serviço Especializado em & 1 Médico do trabalho & 3 Técnicos de segurança do trabalho \\
\hline Engenharia de Segurança e & 3 Técnicos de segurança do trabalho & Departamento de SST subordinado \\
\hline Medicina do Trabalho) & $\begin{array}{l}\text { Departamento de SST subordinado } \\
\text { diretamente à alta direção }\end{array}$ & diretamente à alta direção \\
\hline Certificações & $\begin{array}{l}\text { ISO } 9001 \text { (Gestão da qualidade) e } \\
\text { ISO } 14001 \text { (Gestão ambiental) }\end{array}$ & ISO 9001 (Gestão da qualidade) \\
\hline Política formal de SST & Não possui & $\begin{array}{l}\text { A segurança é mencionada na } \\
\text { política da qualidade }\end{array}$ \\
\hline Investimentos em SST & $\begin{array}{l}\text { Investimentos em Equipamentos } \\
\text { de Proteção Individual (EPIs), } \\
\text { Equipamentos de Proteção Coletiva } \\
\text { (EPCs) e treinamentos (técnicos) }\end{array}$ & $\begin{array}{l}\text { Investimentos em EPIs, EPCs } \\
\text { e treinamentos (técnicos e de } \\
\text { conscientização: motivacionais e de } \\
\text { gestão) }\end{array}$ \\
\hline Alta direção & $\begin{array}{l}\text { Investe em melhorias na SST, porém } \\
\text { não a considera uma prioridade }\end{array}$ & $\begin{array}{l}\text { Investe em SST e participa ativamente } \\
\text { do planejamento de ações e análise de } \\
\text { resultados }\end{array}$ \\
\hline Programas de SST & $\begin{array}{l}\text { Possui os programas exigidos por } \\
\text { lei, como o Programa de Prevenção } \\
\text { de Riscos Ambientais (PPRA), o } \\
\text { Programa de Controle Médico de } \\
\text { Saúde Ocupacional (PCMSO) e Mapa } \\
\text { de Riscos }\end{array}$ & $\begin{array}{l}\text { Possui o PPRA, o PCMSO, o } \\
\text { mapa de riscos e mantém um programa } \\
\text { de alguns indicadores relativos à SST }\end{array}$ \\
\hline $\begin{array}{l}\text { Comunicação Interna } \\
\text { relacionada à SST }\end{array}$ & $\begin{array}{l}\text { Realiza uma reunião semanal } \\
\text { sobre SST (Diálogo Semanal de } \\
\text { Segurança - DSS) em que são relatados } \\
\text { aos trabalhadores os acidentes } \\
\text { ocorridos e as ações corretivas e } \\
\text { preventivas tomadas } \\
\text { Pouco investimento em ferramentas de } \\
\text { comunicação interna }\end{array}$ & $\begin{array}{l}\text { Realiza reuniões sobre SST apenas } \\
\text { quando julga conveniente } \\
\text { A comunicação interna se dá por } \\
\text { meio de painéis de aviso e banners } \\
\text { espalhados pela fábrica }\end{array}$ \\
\hline Recursos humanos & $\begin{array}{l}\text { Baixo envolvimento da área de } \\
\text { recursos humanos em treinamentos } \\
\text { relacionados à SST } \\
\text { Está iniciando um programa de } \\
\text { desenvolvimento de lideranças que } \\
\text { auxiliará diretamente o SGSST }\end{array}$ & $\begin{array}{l}\text { O RH participa consideravelmente das } \\
\text { ações realizadas pelo Departamento de } \\
\text { Segurança, principalmente no que diz } \\
\text { respeito a treinamento de funcionários, } \\
\text { transferências para outro setor ou } \\
\text { função e orientações gerais }\end{array}$ \\
\hline Treinamentos relacionados à SST & $\begin{array}{l}\text { Possui uma programação formal } \\
\text { de treinamento sobre SST. Os } \\
\text { treinamentos são realizados, no } \\
\text { mínimo, uma vez ao ano }\end{array}$ & $\begin{array}{l}\text { Também adota a política de } \\
\text { treinamentos anuais }\end{array}$ \\
\hline $\begin{array}{l}\text { Medição e análise de } \\
\text { desempenho em SST }\end{array}$ & $\begin{array}{l}\text { Não possui indicadores de desempenho } \\
\text { de SST. Todas as ocorrências e } \\
\text { ações são divulgadas nos DDSs e } \\
\text { nas reuniões gerenciais, em que são } \\
\text { discutidas as ações realizadas }\end{array}$ & $\begin{array}{l}\text { A organização possui indicadores } \\
\text { de acompanhamento dos níveis de } \\
\text { chumbo de seus funcionários, faz } \\
\text { levantamento dos riscos predominantes } \\
\text { em cada setor e a classificação das } \\
\text { principais ocorrências }\end{array}$ \\
\hline $\begin{array}{l}\text { Medidas corretivas e } \\
\text { preventivas de SST }\end{array}$ & $\begin{array}{l}\text { Possui em suas instalações } \\
\text { Equipamentos de Proteção Coletiva } \\
\text { (EPCs) e exige que seus colaboradores } \\
\text { usem EPIs } \\
\text { Os problemas detectados são } \\
\text { corrigidos por meio da participação } \\
\text { dos principais envolvidos }\end{array}$ & $\begin{array}{l}\text { Os técnicos de segurança e membros } \\
\text { da CIPA realizam inspeções no local } \\
\text { de trabalho } \\
\text { As não conformidades são } \\
\text { encaminhadas ao setor responsável por } \\
\text { sua correção }\end{array}$ \\
\hline Programas de incentivo à SST & Não há & Não há \\
\hline
\end{tabular}


Quadro 2. Principais dificuldades encontradas no gerenciamento do SGSST na Empresa 1 e Empresa 2.

\begin{tabular}{|c|c|}
\hline \multicolumn{2}{|c|}{ Principais dificuldades do gerenciamento da SST nas empresas estudadas } \\
\hline Empresa 1 & Empresa 2 \\
\hline Resistência à mudança & Resistência à mudança \\
\hline Dificuldade na elaboração de procedimentos e instruções & Conflitos internos \\
\hline Falhas na comunicação interna & $\begin{array}{l}\text { Falhas na comunicação dos objetivos a serem alcançados } \\
\text { com a implantação de novos sistemas de SST }\end{array}$ \\
\hline $\begin{array}{l}\text { Baixo envolvimento dos setores da organização, que } \\
\text { entendem que o Departamento de Segurança é o único } \\
\text { responsável pelos resultados do SGSST }\end{array}$ & $\begin{array}{l}\text { Temor das auditorias devido à insegurança e medo de } \\
\text { errar }\end{array}$ \\
\hline $\begin{array}{l}\text { Ausência de indicadores de desempenho em segurança e } \\
\text { saúde no trabalho }\end{array}$ & Alta rotatividade de funcionários terceirizados \\
\hline Falta de comprometimento da média gerência & $\begin{array}{l}\text { Dificuldades em se compreender o processo de } \\
\text { implantação de sistemas de SST }\end{array}$ \\
\hline $\begin{array}{l}\text { Baixo índice de conscientização dos funcionários com } \\
\text { relação à SST }\end{array}$ & Recursos financeiros insuficientes para a SST \\
\hline
\end{tabular}

\subsection{Estratégia organizacional}

O planejamento estratégico cabe à alta direção, que tem a responsabilidade de formular os objetivos maiores da organização baseados na missão e visão da empresa. Ele tem como uma das funções apoiar os programas de SST por meio do planejamento e disponibilização de recursos técnicos, humanos e financeiros.

Os objetivos e metas relativos à SST definidos pela alta direção devem ser entendidos por todos e desdobrados no planejamento tático e operacional, no qual as metas são estabelecidas de acordo com as necessidades dos diversos níveis da organização. A inserção da SST na estratégia organizacional permite vislumbrar que o aporte financeiro e humano necessário para seu bom desempenho será fornecido, facilitando muito o desenvolvimento e implantação de um SGSST.

\subsection{Cultura organizacional}

Uma cultura de segurança sólida pode ser construída ou modificada a partir do entendimento da diversidade e complexidade organizacional. A cultura organizacional quando bem gerenciada pode ser utilizada a favor do fortalecimento da política de segurança e saúde no trabalho e, consequentemente, do desenvolvimento de SGSSTs.

Fazer uma análise prévia do perfil dos colaboradores, identificando o grau de escolaridade, o seu comportamento na execução das atividades, a sua participação na sugestão de melhorias e o seu relacionamento com as lideranças e pares são fatores importantes para que se consiga, antecipadamente, identificar as principais resistências que podem surgir durante o processo de implantação do SGSST.

Os dados obtidos por meio deste levantamento podem ajudar na elaboração de programas de treinamento e conscientização e influenciar positivamente atitudes e habilidades dos colaboradores, que colaborarão mais facilmente para que a organização atinja seus objetivos em relação à SST.

\subsection{Departamento de Segurança e Saúde no Trabalho}

A subordinação do Departamento de Segurança e Saúde do Trabalho diretamente à alta direção é um aspecto importante para que os objetivos de SST sejam mais eficazmente alcançados, inclusive o desenvolvimento de um SGSST.

Este contato direto faz com que o Departamento de Segurança e Saúde do Trabalho tenha maior autonomia na discussão e planejamento dos projetos que envolvam a segurança e a saúde do trabalhador.

As ações de segurança, lideradas por este Departamento, devem envolver todos os setores da organização. Esta característica é importante porque possibilita que tais ações sejam desenvolvidas com visões variadas e com equipes multidisciplinares, evitando prejuízos devido a interesses individuais de cada setor.

Os demais setores devem estar conscientes de que o desempenho da SST está sendo diretamente acompanhado pela alta direção, que tem a responsabilidade de definir objetivos e cobrar resultados.

\subsection{Técnicos de Segurança e Saúde do Trabalho}

O perfil dos profissionais responsáveis por liderar as iniciativas de SST na empresa é um fator de extrema importância para que o projeto de desenvolvimento de um SGSST tenha sucesso. Além do conhecimento técnico, é necessário que eles possuam determinadas 
habilidades como comunicação e relacionamento interpessoal, elementos fundamentais para liderar grupos. Deve-se investir intensamente em seu treinamento, pois esta é uma forma de aumentar a eficiência e a eficácia do SGSST.

A habilidade em comunicação não envolve somente a capacidade de disseminar a informação em forma verbal ou escrita, mas ser capaz de transformá-la em linguagem compreensível a todos.

Eles devem desenvolver habilidades para resolver problemas e detectar oportunidades de melhorias, buscando sempre a participação efetiva dos colaboradores. Devem evitar a punição, que pode causar resistências e desentendimentos, e priorizar a conscientização, a motivação e a recompensa.

\subsection{Recursos humanos}

É importante que todos os setores da organização desenvolvam trabalhos em conjunto com a área de RH, pois esta integração entre departamentos gera resultados positivos para toda a empresa e possibilita a construção de um ambiente interno com menos conflitos e resistências.

O envolvimento da área de RH na implantação do SGSST é importante para que sejam identificadas as necessidades de treinamentos técnicos e de desenvolvimento pessoal/gerencial, possibilitando melhor entendimento e real comprometimento dos colaboradores com as mudanças propostas.

A área de recursos humanos pode realizar a identificação das competências e habilidades necessárias para cada função e treinar os colaboradores para o alcance dos objetivos de SST. O importante é que a equipe que lidera o desenvolvimento e implantação do SGSST trabalhe em conjunto com a área de RH. Esta parceria permitirá que focos de resistência sejam mais bem identificados e resolvidos antes do projeto ser efetivamente levado a cabo, que os treinamentos sejam personalizados e que os aspectos da cultura organizacional sejam utilizados a favor do SGSST.

\subsection{Treinamento}

O treinamento deve ser entendido como um meio estratégico de desenvolver competências para que os colaboradores se tornem mais produtivos, criativos, inovadores e possam contribuir para o alcance dos objetivos organizacionais, incluindo aqueles relativos à SST. Para isto, é necessário que sejam planejados de acordo com as necessidades identificadas e de acordo com os requisitos estabelecidos para cada função.

$\mathrm{O}$ treinamento deve-se focar tanto no desenvolvimento do conhecimento técnico como nas características pessoais. No caso da implantação de SGSSTs, o treinamento ganha uma dimensão ainda mais importante, pois é a partir dele que se dá a grande oportunidade de mobilização da mão de obra para a questão da segurança.

Os treinamentos podem ser realizados internamente ou por empresas e profissionais terceirizados. Em ambas as situações devem ser realizados em parceria estreita com os profissionais de recursos humanos que, utilizando as ferramentas de conscientização e motivação adequadas, podem ajudar na obtenção de melhores resultados. O treinamento é uma importante "arma" para viabilizar o desenvolvimento e implantação de SGSSTs.

\subsection{Equipes multidisciplinares}

As equipes multidisciplinares são compostas por membros de diversas áreas e com os mais variados conhecimentos e podem contribuir para que o SGSST alcance os resultados esperados. No caso da segurança do trabalho, pode-se considerar a CIPA como um importante exemplo de uma equipe multidisciplinar.

Seus integrantes são responsáveis por fiscalizar se os procedimentos de segurança estão sendo cumpridos e propor soluções de melhoria para os problemas relacionados, além de auxiliarem na estruturação de uma eventual implantação de SGSST.

Estas averiguações podem ser formais (periódicas e planejadas) ou informais, quando um membro da equipe detecta que um trabalhador está realizando um ato inseguro ou que o local de trabalho apresenta uma condição insegura. Nestes casos, o trabalhador deve ser imediatamente conscientizado quanto ao risco e o fato comunicado aos técnicos de segurança, para que seja devidamente registrado e as providências para sua não ocorrência tomadas.

Para que haja centralização das informações e mais rapidez na solução dos problemas, todos os dados colhidos pela equipe de SST ou pelos chefes dos diversos setores nestas circunstâncias deverão ser comunicados ao Departamento de Segurança e Saúde do Trabalho em impresso próprio o mais breve possível.

\subsection{Comunicação interna}

A empresa deve utilizar os meios e técnicas de comunicação compatíveis com o nível de cultura de seus funcionários (quadros de avisos, intranet, treinamentos, reuniões, jornais internos, etc.), a fim de informar a todos sobre os objetivos da empresa e as oportunidades que novos procedimentos ou um novo sistema de gestão da segurança podem proporcionar.

O papel da comunicação é importante para o esclarecimento de novos programas e suas particularidades e deve ser feita de maneira clara, 
concisa e, dentro do possível, sem muitos termos complexos, utilizando linguagem adaptada à realidade dos colaboradores para que todos possam entender com precisão o que está sendo informado.

Deve-se verificar se as comunicações foram entendidas claramente e, em caso negativo, é imprescindível repeti-las com as modificações necessárias para que se garanta sua eficácia, quantas vezes forem precisas, buscando sempre o feedback dos receptores para seu contínuo aprimoramento.

Se este processo for executado de forma satisfatória, será estabelecido alto nível de entendimento e comprometimento entre a empresa e seu quadro de funcionários, reduzindo boatos capazes de gerar temores e transtornos contraproducentes.

\subsection{Resistência à mudança}

Fenômenos como conflitos, incertezas, medo do desconhecido, falta de informação e sensações de perda de poder podem gerar resistência às mudanças e interferir negativamente na implantação de SGSSTs nas organizações.

Os trabalhadores precisam estar conscientes de que um processo de mudança traz novas oportunidades, como o desenvolvimento de habilidades, motivação para a busca de novos desafios e possibilidade de desenvolvimento profissional.

Devem ser desenvolvidas e utilizadas as ferramentas de gestão disponíveis para minimizar tas resistências, auxiliadas pelo estabelecimento de incentivos à participação de todos no processo de implantação do SGSST, a partir da valorização de opiniões, intensa capacitação e adequada disponibilização de informações. É interessante se criar um programa estruturado para fomento de sugestões de melhorias em relação à SST, de forma que os colaboradores se sintam à vontade e motivados a colaborar.

A participação efetiva dos agentes de mudança é muito importante nos processos de implantação do SGSST. Os trabalhadores precisam se sentir seguros quanto ao comprometimento de seus líderes, conhecerem os objetivos da organização e sua relação com a SST e qual a sua efetiva participação e importância neste processo, pois somente assim se sentirão responsáveis e se envolverão seriamente.

\subsection{Indicadores de desempenho}

É preciso definir, em trabalho conjunto com todas as áreas da organização, indicadores de desempenho para SST de forma que se possa monitorar o desempenho do SGSST e identificar oportunidades de melhorias. Quantos e quais indicadores são decisões que vão depender das características de cada empresa, considerando seu porte, tipo de atividade, graus de risco a que está submetida, etc.
Os indicadores têm como objetivo permitir que toda empresa, e principalmente a alta direção, visualizem o desempenho do SGSST como um todo, possibilitando a realização de uma autoavaliação e o estabelecimento de planos para eventuais correções de rumos. Eles devem ser pensados desde o planejamento do sistema, antes de sua implantação efetiva.

É importante que os indicadores sejam avalizados pela alta cúpula da empresa e forneçam informações capazes de apoiar não só o SGSST, mas também o plano estratégico organizacional. Eles devem estar acessíveis e ser de conhecimento de todos os colaboradores, que precisam saber interpretá-los e conhecerem as ações estabelecidas a partir deles para evitar ou mitigar acidentes.

\subsection{Ferramentas gerenciais para a solução de problemas de Segurança e Saúde do Trabalho}

Capacitar a equipe de segurança em ferramentas gerenciais facilita os processos de planejamento, organização, classificação, priorização, análise e solução de problemas de SST. Estas ferramentas possibilitam que os envolvidos trabalhem em equipe, por meio da troca produtiva de ideias e sugestões de maneira muito mais eficaz, facilitando a implantação de sistemas deste tipo.

Pode-se capacitar os colaboradores-chave tanto internamente (com multiplicadores) como externamente (empresas de treinamento especializadas) com base na filosofia de "círculos de controle da qualidade" ou "times da qualidade", porém adaptada à realidade da SST.

As ferramentas mais comuns para serem utilizadas por estes grupos são: brainstorming, benchmarking, círculo PDCA (plan, do, check e act), ferramentas estatísticas, FMEA (Failure Mode and Effect Analysis), diagrama de causa e efeito (diagrama de Ishikawa), $5 \mathrm{~W} 2 \mathrm{H}$ (ferramenta para desenvolvimento de planos de ação: what?, why?, where?, who?, when?, how? e how much?), etc.

\subsection{Gestão de projetos}

A utilização da metodologia de gestão de projetos para a implantação de SGSSTs tem como objetivo auxiliar a organização na gestão de aspectos referentes ao planejamento, execução e controle das atividades relacionadas ao novo projeto.

Para sua utilização é necessário que a organização estabeleça objetivos precisos quanto aos resultados esperados com o projeto de SST e determine os principais responsáveis para sua execução e acompanhamento. Estes procedimentos permitem, muitas vezes, a identificação antecipada dos problemas e possibilita a realização de ações preventivas. 
Como vantagens, a metodologia de gestão de projetos permite um ciclo menor do processo de implantação do SGSST, custos menores, decisões mais eficazes e menos improvisos. Contudo, para que o gerenciamento de projetos seja bem-sucedido é necessário que a organização invista tempo e esforço na realização do planejamento do sistema.

Esta metodologia, além de permitir um acompanhamento detalhado de cada fase, estimula o trabalho em equipe, a busca por resultados e possibilita o levantamento e arquivamento de informações que podem ser utilizadas em futuros projetos. Aconselha-se que a gestão do projeto de implantação de um SGSST seja baseado nos preceitos do PMBOK (Project Management Body of Knowledge) com auxílio das ferramentas PERT/CPM (Program Evaluation and Review Technique/Critical Path Method).

\subsection{Recompensas e incentivos}

A utilização de um sistema de recompensa e incentivos, tanto durante o processo de implantação como de gerenciamento rotineiro de um SGSTT, faz com que os colaboradores se sintam mais motivados a aprender e a buscar soluções que melhorem o desempenho do sistema.

A recompensa e o incentivo aos profissionais e setores que se destacaram na SST (maior número de ideias sugeridas, melhores indicadores em SST, etc.) em determinado período podem se dar de várias formas: destaque (quadros com fotos, jornais internos, citação em reuniões, etc.), viagens, prêmios (como um eletrodoméstico, por exemplo) e recompensa monetária.

A empresa pode avaliar o desempenho do seu SGSST por meio de indicadores de desempenho individuais e coletivos (setoriais) juntamente com os resultados das inspeções realizadas pelos membros da CIPA, técnicos de segurança, auditorias, etc.

A concessão de recompensas produz motivação em toda a equipe, porém, deve-se ter cuidado para que os atos inseguros e os acidentes não sejam ocultados com o objetivo de demonstrar bons resultados e também para que o sistema de premiação não se transforme em uma forma de competição não sadia entre os setores da empresa.

\subsection{Integração do sistema}

Em geral, um SGSST certificável é desenvolvido e implantado simultaneamente com sistemas da qualidade e meio ambiente ou em empresas que já os possuam. Em qualquer destas situações a integração destes sistemas, que possuem as mesmas bases (ciclo PDCA, melhoria contínua e padronização), é fundamental para se evitarem duplicações de recursos, burocracia desnecessária, desmotivação de colaboradores e para possibilitar ganhos sinérgicos.
Esta integração contribui para a melhoria da eficiência e eficácia das organizações, pois permite o alinhamento de objetivos, processos e recursos e pode se dar de várias maneiras: documentos únicos para instruções de trabalho, treinamentos conjuntos, avaliação simultânea dos sistemas, auditorias partilhadas, etc.

\section{Considerações finais}

Acredita-se que a apresentação das diretrizes constantes do item 5 deste trabalho, que foram elaboradas com base no referencial teórico e nos resultados dos dois estudos de caso, possibilitou o cumprimento do objetivo estabelecido.

Estas diretrizes são a sistematização das recomendações identificadas na teoria, somadas as boas práticas verificadas nos estudos de caso com a consideração também dos seus contraexemplos (dificuldades) na implantação de SGSSTs. Espera-se que elas possam, ainda que tenham características genéricas, efetivamente cumprir um papel orientativo para que as empresas produtoras de baterias automotivas desenvolvam e implantem sistemas deste tipo com menos dificuldades, potencializando também seus resultados.

Apesar das diretrizes aqui apresentadas terem sido desenvolvidas considerando-se o estudo de duas empresas de baterias automotivas, em função de sua elaboração ter sido também fortemente baseada no referencial teórico - que é genérico -, é possível que, com bastante cuidado e considerando as características do ambiente externo, a cultura organizacional e as peculiaridades, se possa adaptá-las a outros tipos de empresas industriais.

A pesquisa qualitativa com base no método de estudo de caso mostrou-se apropriada a este trabalho, pois possibilitou que a realidade e as principais características das empresas de baterias estudadas fossem devidamente interpretadas e compreendidas, auxiliando na formulação das diretrizes propostas.

Procurou-se apresentar neste artigo um referencial teórico que contemplasse e subsidiasse a realização dos estudos de caso e formulação das diretrizes para implantação de SGSSTs em empresas do setor de baterias automotivas.

Cabe destacar as limitações desta pesquisa quanto à impossibilidade de generalização estatística dos seus resultados, quanto à concentração das empresas estudadas em uma única região (Bauru) e quanto ao porte das empresas estudadas (apenas de médio porte).

\section{Referências}

AGGELOGIANNOPOULOS, D.; DROSINOS, E. H.; ATHANASOPOULOS, P. Implementation of a quality management system (QMS) according to the ISO 9000 family in a Greek small-sized winery: a case study. Food Control, v. 18, n. 9, p. 1077-1085, 2007. 
AKSORN, T.; HADIKUSUMO, R. H. W. Critical success factors influencing safety program performance in Thai construction projects. Safety Science Journal, v. 46, n. 4, p. 709-727, 2008.

ANHALON, R.; ZOQUI, E. J.; PINTO, J. S. Principais dificuldades vivenciadas na implementação de um sistema de gestão da qualidade adaptado em empresas incubadas na região de Jundiaí. In: ENCONTRO NACIONAL DE ENGENHARIA DE PRODUÇÃO, XXIV, 2005, Porto Alegre. Anais... Porto Alegre: ABREPO, 2005.

BOSCH. O que é uma bateria? 2008. Disponível em: <http://www.bosch.com.br/br/autopecas/produtos/ baterias/o_que_e.htm >. Acesso em: 15 mar. 2010.

CENTRO DA QUALIDADE, SEGURANÇA E PRODUTIVIDADE - QSP. OHSAS 18001: sistemas de gestão da segurança e saúde no trabalho. Disponível em <http://www.qsp.org.br/frame.php?p=recertificacao. shtml.>. Acesso em: 30 set. 2007.

CERVO, A. L.; BERVIAN, P. A. Metodologia científica. São Paulo: Prentice-Hall, 2002.

CHAN, E. S. W.; WONG, S. C. K. Motivations for ISO 14001 in the hotel industry. Tourism Management, v. 27, n. 3, p. 481-492, 2006.

CHOUDHRY, R. M.; FANG, D.; MOHAMED, S. The nature of safety culture: a survey of the state-of-the-art. Safety Science Journal, v. 45, n. 10, p. 993-1012, 2007.

DINGSDAG, D. P.; BIGGS, H. C.; SHEAHAN, V. Understanding and defining $\mathrm{OH} \& \mathrm{~S}$ competency for construction site positions: worker perceptions. Safety Science Journal, v. 46, n. 4, p. 619-633, 2008.

DUIJIM, N. J. et al. Management of health, safety and environment in process industry. Safety Science Journal, v. 46, n. 6, p. 908-920, 2008.

GODOY, R. S. P.; PEÇANHA, D. L. A influência da cultura organizacional nos processos de inovação: uma revisão da literatura. In: SIMPÓSIO DE ENGENHARIA DE PRODUÇÃO, XIV, 2007, Bauru. Anais eletrônicos... Bauru: UNESP, 2007. Disponível em: <http://www. simpep.feb.unesp.br/>. Acesso em: $10 \mathrm{dez} .2007$.

HASLAM, R. A. et al. Contributing factors in construction accidents. Applied Ergonomics Journal, v. 36, n. 4, p. 401-415, 2005.

HASLE, P.; JENSEN, P. L. Changing the internal health and safety organization through organizational learning and change management. Human Factors and Ergonomics in Manufacturing, v. 16, n. 3, p. 269-284, 2006.

LAKATOS, E. M.; MARCONI, M. A. Técnicas de pesquisa. São Paulo: Atlas, 2002.

LANGFORD, D.; ROWLINSON, S.; SAWACHA, E. Safety behavior and safety management: its influence on the attitudes in the UK construction industry. Engineering Construction and Architectural Management Journal, v. 7, n. 2, p. 133-140, 2000.

LOOSEMORE, M; ANDONAKIS, N. Barriers to implementing OHS reforms: the experiences of small subcontractors in the Australian Construction Industry. Journal of Project Management, v. 25, n. 6, p. 579-588, 2007.

MARQUEZE, E. C.; MORENO, C. R. C. Satisfação no trabalho: uma breve revisão. Revista Brasileira de Saúde Ocupacional, v. 30, n. 112, p. 67-79, 2005.
MATTOS, U. A. O. et al. Avaliação e diagnóstico das condições de trabalho em duas indústrias de baterias chumbo-ácidas no estado do Rio de Janeiro. Revista Ciência \& Saúde Coletiva, v. 8, n. 4, p. 1047-1056, 2003.

MENEZES, J. A.; CARVALHO, W. A.; SPÍNOLA, A. G. Avaliação da exposição ocupacional ao chumbo em uma metalúrgica: um estudo transversal. Revista Brasileira de Saúde Ocupacional, v. 28, n. 105-106, p. 63-72, 2004.

MILAN, G. S.; PRETTO, M. R.; PIGOZZI, P. R. A relação entre a gestão da qualidade e a cultura organizacional: um estudo de caso ambientado em uma fábrica de embalagens de papelão. In: ENCONTRO NACIONAL DE ENGENHARIA DE PRODUÇÃO, XXIV, 2005, Porto Alegre. Anais... Porto Alegre: ABREPO, 2005.

MILKOVICH, G. T.; BOUDREAU, J. W. Administração de recursos humanos. São Paulo: Atlas, 2000.

OLIVEIRA, J. C. Segurança e saúde no trabalho: uma questão mal compreendida. São Paulo em Perspectiva, v. 17, n. 2, p. 3-12, 2003.

OLIVEIRA, O. J. Gestão da qualidade: introdução à história e fundamentos. In:___ Gestão da qualidade: tópicos avançados. São Paulo: Thomson Learning, 2004.

OHSAS 18001 - Occupational health and safety management systems: requirements. London, 2007

PINTO, E. N. F., SÁ, V.C. A gestão de pessoas e o processo de implantação da OHSAS 18001: um estudo de caso. In: SIMPÓSIO DE ENGENHARIA DE PRODUÇÃO, 2007, Bauru. Anais eletrônicos... Bauru: UNESP, 2007. Disponível em: http://www.simpep.feb.unesp. br/. Acesso em: 10 ago. 2008.

QSP - Centro da qualidade, segurança e produtividade. Sistemas de Gestão da Segurança e Saúde no Trabalho. Disponível em <http://www.qsp.org.br/ frame.php? $\mathrm{p}=$ recertificacao.shtml. $>$ Acesso em: 30 set. 2007

QUELHAS, O. L. G.; ALVES, M. S.; FILARDO, P. S. As práticas da gestão da segurança em obras de pequeno porte: integração com os conceitos de sustentabilidade. Revista Produção OnLine, v. 4, n. 2, 2003. Disponível em: <www.producaoonline.inf.br>. Acesso em: 1 abr. 2007.

QUINTELLA, O. M.; LIMA, G. B. O balanced scorecard como ferramenta para a implantação da estratégia: uma proposta de implantação. In: ENCONTRO NACIONAL DE ENGENHARIA DE PRODUÇÃO, XXIII, 2004, Florianópolis. Anais... Florianópolis: ABREPO, 2004.

RAYMOND, L; BERGERON, F. Project management information systems: an empirical study of their impact on project managers and project success. International Journal of Project Management, v. 26, n. 2, p. 213-220, 2008.

RONDINELLI, D.; VASTAG, G. Panacea, common sense, or just a label? The value of ISO 14001 environmental management systems, 2000. Disponível em: <http://www. ingentaconnect.com/content/els/02632373/2000/00000 018/00000005/art00039>. Acesso em: 11 jan. 2007. 
SANTOS, G. T.; ROSSI, G.; JARDILINO, J. R. L. Orientações metodológicas para elaboração de trabalhos acadêmicos. São Paulo: Gion Editora, 2000.

SBRT - SERVIÇO BRASILEIRO DE RESPOSTAS TÉCNICAS. 2006. Baterias automotivas. Disponível em: <http://www.sbrt.ibict.br/pages/index.jsp;jsessio nid=BC7EFF79ADE3D874D81ECE419DC34C81>. Acesso em: 15 mar. 2008.

SHI, H. et al. Barriers to the implementation of cleaner production in Chinese SMEs: government, industry and expert stakeholders' perspectives. Journal of Cleaner Production, v.16, n. 7, p. 842-852, 2008.

TRIVELATO, G. C. Sistema de gestão da segurança e saúde no trabalho: fundamentos e alternativas. In: SEMINÁRIO NACIONAL SOBRE GESTÃO DA SEGURANÇA E SAÚDE NO TRABALHO, 2002, Belo Horizonte. Anais...: Fundacentro, 2002. Disponível em: < http://www.fundacentro.gov.br/ CTN/sistemas_gestao_saude_trabalho.pdf $>$. Acesso em: 1 abr. 2008.

YIN, R. K. Case study research: design and methods. Newbury Park: Sage Publications, 2003. 